\title{
Conocimiento, prácticas y actitudes alimentarias de vegetarianos en Paraguay
}

\author{
Erika Giselle Centurión-Bernal ${ }^{1}$, Ana Gabriela González-Acosta ${ }^{1}$, María Belén Rojas-Pavón ${ }^{1}$, Rodrigo \\ Octavio Burgos-Larroza1, *Eliana Meza-Miranda² \\ 1. Facultad de Ciencias Químicas, Universidad Nacional de Asunción. Paraguay \\ 2. Centro Multidisciplinario de Investigaciones Tecnológicas, Universidad Nacional de Asunción. Paraguay
}

\section{Cómo referenciar este artículo/ How to reference this article:}

\begin{abstract}
Centurión-Bernal EG, González-Acosta AG, RojasPavón MB, Burgos-Larrosa RO, Meza-Miranda E. Conocimiento, prácticas y actitudes alimentarias de vegetarianos en Paraguay. Mem. Inst. Investig. Cienc. Salud. 2018; 16(1): 19-25
\end{abstract}

\section{RE S U M E N}

El auge de las dietas vegetarianas se encuentra relacionado principalmente a beneficios para la salud, tratamiento de ciertas patologías y la obtención de una dieta equilibrada, a su vez con principios animalistas o morales. El objetivo fue evaluar el conocimiento, describir las prácticas y actitudes alimentarias de vegetarianos en Paraguay entre mayo a julio del 2017. Se realizaron 141 encuestas, de las cuales 132 cumplieron las exigencias del estudio. La mayor parte de los encuestados fue del sexo femenino (79\%), la edad promedio fue de $28,7 \pm 8,1$ años, $93 \%$ de ellos eran del nivel educativo terciario, gran parte de los participantes eran residentes del área central (87\%), su fuente principal de obtención de información eran los profesionales de la salud (12\%) seguidos de internet (11\%). Un 44\% consumía suplementos alimentarios, y la razón principal de haber optado por el estilo de vida vegetariano fueron principios animalistas, morales o de protección animal (51\%). Por otro lado, a un $25 \%$ le resultaba difícil asistir a comidas o reuniones sociales. Los encuestados presentaron conocimiento regular seguido de insuficiente en lo que respecta a su estilo de vida y conocimiento insuficiente sobre nutrientes críticos. Respecto a sus prácticas, gran parte de ellos reemplazaron las proteínas por las de origen vegetal obteniendo información de diversas fuentes. Actitudinalmente creían que su alimentación favorecía a su salud e imagen corporal y la recomendaban, habiendo sufrido discriminación en algún momento de su vida mediante críticas o burlas hacia su estilo de vida.

Palabras clave: vegetarianismo, conocimiento, prácticas, actitudes.

\section{Knowledge, practices and alimentary attitudes of vegetarians in Paraguay}

\section{A B S T R A C T}

The boom of vegetarian diets is mostly related to health benefits, treatment of certain pathologies and a balanced diet, but at the same time to animalism and moral principles. The objective of this study was to evaluate the knowledge and describe practices and alimentary attitudes of vegetarians in Paraguay from May to July 2017. One hundred and forty one questionnaires were collected, 132 complied the study requirements. Most of the respondents were female $(80 \%)$, the mean age was $29 \pm 8.1$ years, $93 \%$ of them has tertiary educational level, most of the respondents were residents of the Central Department $(87 \%)$, and their main information source was health professionals $(12 \%)$ followed by the use of internet ( $11 \%)$. Among them, $44 \%$ consume alimentary supplements while the main reasons to opt for a vegetarian lifestyle were animalism and moral principles $(51 \%)$. On the other hand, $25 \%$ found difficult to attend meals and social meetings. The surveyed vegetarians mostly presented a regular knowledge followed by insufficient with regard to their lifestyle and an insufficient knowledge concerning critical nutrients. In 
relation to their practices, a lot of them replaced animal proteins for those of vegetal origin obtaining information from diverse sources. Attitudinally they believed that their diet favored their health and corporal image, and therefore they recommended it though they had suffered discrimination in some moment of their lives through criticism and jibes about their lifestyle.

Keywords: vegetarianism, knowledge, practices, attitudes.

\section{INTRODUCCIÓN}

En la actualidad existen varios estilos de vida que incluyen diferentes tipos de alimentación según la elección o preferencia de las personas. Una de ellas es la omnívora, la cual no implica restricciones en el consumo, es decir que incluye a todos los grupos de alimentos; cumple con las leyes de la alimentación permitiendo mantener un óptimo estado de salud, cubre las necesidades nutricionales para el desarrollo y conservación del organismo y por sobre todo responde a conceptos de suficiencia, equilibrio, variedad y adaptación ${ }^{(1)}$. Por otro lado, y en contrapartida existen el vegetarianismo y veganismo que son estilos de vida en los cuales se excluyen los alimentos de origen animal y sus derivados siendo más o menos estrictos dependiendo de la corriente a la que pertenezcan ${ }^{(2)}$.

Una de las diferencias entre estos estilos de vida es la reducción de la mortalidad en aquellas personas que llevan un patrón dietario en el que tienen en común una alta ingesta de alimentos de origen vegetal a diferencia de aquellos en los que hay preferencia en el consumo de carnes $^{(3)}$. Particularmente dentro del vegetarianismo, se han establecido múltiples variantes, dentro de las cuales las más practicadas son el veganismo en el que se sigue una dieta exclusivamente basada en alimentos de origen vegetal, excluyendo el consumo de huevos y lácteos, el ovo-vegetarianismo que es una alimentación similar, pero con la inclusión de huevo, el lacto-vegetarianismo en el que se incluyen lácteos y sus derivados, y el lacto-ovo-vegetarianismo incluye huevos, lácteos y sus derivados ${ }^{(2,4)}$.

El auge de las dietas vegetarianas en los últimos años se vio influenciado por consideraciones éticas, cuestiones medio ambientales, factores religiosos y problemas de salud. Así como las dietas vegetarianas, las veganas han ganado reconocimiento y popularidad como una elección dietaria saludable y potencialmente terapéutica ${ }^{(5)}$. No obstante, en la actualidad no se ha difundido suficiente información acerca de la relación existente entre estos estilos de vida y las secuelas a corto o largo plazo ya que su práctica supone la supresión de ingesta de nutrientes esenciales para el organismo ${ }^{(6)}$.

Existen evidencias de que la eliminación de la carne roja se ha asociado a una disminución del riesgo de desarrollar enfermedades cardiovasculares mejorando conjuntamente el estilo de vida, este efecto ha sido vinculado al mayor consumo de verduras, frutas, granos enteros, legumbres y frutos secos; y a un menor consumo de alimentos procesados a refinados ${ }^{(6,7)}$.

El consumo moderado de carnes se considera saludable, mientras que se advierte que un alto consumo conlleva a riesgos para la salud a largo plazo, pues aporta grandes cantidades de grasa al organismo y en ocasiones otros elementos potencialmente dañinos como gérmenes $\mathrm{y}$ aditivos hormonales ${ }^{(8)}$. Entre otros factores positivos se mencionan que la exclusión de la carne roja de la dieta favorece la disminución del índice de masa corporal y de los valores de colesterol plasmático total así como de la mortalidad por infarto cardiaco en comparación con los no vegetarianos ${ }^{(6)}$.

Actualmente esta corriente alimenticia se ha expandido mundialmente, lo cual puede atribuirse a factores asociados a la salud, sociales o ambientales. Aunque la población vegetariana en varias ciudades continúa siendo minoritaria, según evidencias estadísticas la excepción es India, en donde aproximadamente un tercio de la población es vegetariana. Los datos refieren que alrededor del $10 \%$ de los vegetarianos son veganos; lo cual refleja que el número de veganos está aumentando más rápidamente que el de los vegetarianos ${ }^{(9)}$. Asimismo, se estima que un $5 \%$ de la población inglesa es vegetariana, mientras que encuestas masivas en Estados Unidos reportan que un 1,4\% de su población es vegana ${ }^{(4)}$.

Viendo la necesidad de contar con información sobre las condiciones del estilo de vida vegetariano en Paraguay, debido a que existe escasa información referente al tema; el presente estudio tiene como objetivo evaluar los conocimientos, obtener datos sobre cuál de las prácticas es más frecuente y las razones que los motiva a adoptar este estilo de vida. 


\section{MATERIALES Y MÉTODOS}

Estudio observacional, descriptivo, transversal en el que la población objetivo incluyó adultos vegetarianos de ambos sexos entre 18 y 60 años, residentes en Paraguay. El tipo de muestreo fue por conveniencia. Se respetaron los principios éticos de justicia, respeto y beneficio. El protocolo fue aprobado por el Comité de Ética de Investigación de la Facultad de Ciencias Químicas (CEI Nro. 324/17). Para garantizar la participación se realizó una reunión informativa con los representantes de los grupos o sociedades. Todos los que aceptaron y cumplieron con los criterios de inclusión, recibieron la hoja de información, hoja de recomendaciones y llenaron el consentimiento informado.

La recolección de información se realizó mediante un cuestionario diseñado en Word 2010, que incluía 34 preguntas cerradas y abiertas; que constaba de datos demográficos y 19 preguntas sobre conocimientos, 3 de prácticas y 6 de actitudes. El cuestionario en formato on-line fue diseñado en un formulario en el sitio https: //accounts.google.com, que fue difundido por redes sociales, correos electrónicos, blogs entre otros mediante grupos o sociedades de vegetarianos y estuvo disponible a través del enlace https://docs.google.com/forms/d/1Ym_cca_RYav9pzj2IDS9j_CFaPIIhLLFOOMBL6IKxQ/edit.

El tamaño de muestra se estimó a partir del estadígrafo para estudios descriptivos de variable dicotómico de una población finita, con una proporción esperada de 0,50; un nivel de confianza de $95 \%$ y una amplitud de 0,20. La muestra mínima resultante fue de 96 participantes.

Los datos fueron digitalizados, codificados y analizados en el programa informático Microsoft ${ }^{\circledR}$ Office Excel 2010. Se empleó estadística descriptiva para el resumen de los datos. La evaluación del conocimiento se realizó utilizando una escala del 60\%: menos o igual al $60 \%$ de respuestas correctas se consideraron como conocimiento insuficiente, entre $61-80 \%$ de respuestas correctas se consideraron como conocimiento regular y las respuestas correctas mayor a $80 \%$ se consideraron como conocimiento suficiente ${ }^{10-13}$.

\section{RESULTADOS}

Se recibió un total de 141 encuestas, de las cuales se excluyeron 9 por presentar datos incompletos, quedando 132 participantes. El 80\% era del sexo femenino; la edad promedio fue de $28,7 \pm 8,1$, el lugar de residencia más frecuente fue el departamento Central $(87,12 \%)$; el nivel educativo terciario se observó en el $93,18 \%$ y la ocupación de estudiante en el $34,09 \%$ de los participantes (Tabla 1 ).

Tabla 1. Datos sociodemográficos de los participantes. $n=132$

\begin{tabular}{|c|c|c|}
\hline Datos sociodemográficos & $\mathbf{n}$ & $\%$ \\
\hline \multicolumn{3}{|l|}{ Sexo } \\
\hline Femenino & 105 & 79,6 \\
\hline Masculino & 27 & 20,4 \\
\hline \multicolumn{3}{|l|}{ Edad (años) } \\
\hline$\leq 20$ & 14 & 10,6 \\
\hline $21-40$ & 105 & 79,5 \\
\hline $41-60$ & 13 & 9,8 \\
\hline Promediot Desv. Est & $28,7 \pm 8,1($ mediana $=26,5)$ & Máximo-mínimo (60-18) \\
\hline \multicolumn{3}{|l|}{ Nivel educativo } \\
\hline Primario (10 a 90 grado) & 3 & 2,3 \\
\hline Secundario (1er al 3er año) & 6 & 4,5 \\
\hline Terciario (universitario) & 123 & 93,2 \\
\hline \multicolumn{3}{|l|}{ Lugar de residencia } \\
\hline Central & 115 & 87,12 \\
\hline Itapúa & 8 & 6,1 \\
\hline Alto Paraná & 5 & 3,8 \\
\hline Cordillera & 2 & 1,5 \\
\hline Guairá & 1 & 0,8 \\
\hline Misiones & 1 & 0,8 \\
\hline \multicolumn{3}{|l|}{ Ocupación } \\
\hline Estudiante & 45 & 34,1 \\
\hline Trabajador & 37 & 28,0 \\
\hline Profesional de la Salud & 21 & 15,9 \\
\hline Ama de casa & 4 & 3,0 \\
\hline Otros & 25 & 18,9 \\
\hline
\end{tabular}


Se evaluaron los conocimientos de nutrientes críticos con base en 12 preguntas referentes a las fuentes de los nutrientes críticos y posibles consecuencias que se ven asociados con su bajo aporte, de los cuales un 40,9\% presentaba conocimiento insuficiente. El conocimiento sobre el estilo de vida vegetariano fue indagado en base a 19 preguntas, 17 de las cuales fueron evaluadas. Se obtuvo entre los vegetarianos $35,6 \%$ de conocimiento regular y $30,3 \%$ de conocimiento suficiente (Tabla 2 ).

Tabla 2. Evaluación sobre conocimiento de nutrientes críticos y estilo de vida vegetariano. $n=132$

\begin{tabular}{lll}
\hline Nivel de conocimientos & $\mathbf{n}$ & $\mathbf{\%}$ \\
\hline Sobre nutrientes críticos & & \\
$\quad$ Suficiente $(\geq 81 \%)$ & 40 & 30,3 \\
$\quad$ Regular $(61-80 \%)$ & 38 & 28,8 \\
$\quad$ Insuficiente $(\leq 60 \%)$ & 54 & $\mathbf{4 0 , 9}$ \\
Sobre el estilo de vida vegetariano & & \\
$\quad$ Suficiente $(\geq 81 \%)$ & 40 & 30,3 \\
$\quad$ Regular $(61-80 \%)$ & 47 & $\mathbf{3 5 , 6}$ \\
Insuficiente $(\leq 60 \%)$ & 45 & 34,1 \\
Total & $\mathbf{1 3 2}$ & $\mathbf{1 0 0 \%}$ \\
\hline
\end{tabular}

En lo referente a reemplazar las proteínas de origen animal por otras fuentes proteicas un $90,15 \%$ sostuvo optar por sustituirlas. La pregunta sobre la fuente principal de donde obtienen la información nutricional dio a conocer que el $14 \%$ acudía a varias fuentes dentro de ellas nutricionistas u otros profesionales de la salud, círculo familiar y/o social, internet, ser autodidacta, utilizar libros, radio, televisión y/o revistas; en este ítem los encuestados tuvieron la posibilidad de seleccionar más de una opción. Entre las categorías individuales reflejó que un $12,12 \%$ acudía a nutricionistas, un $11,36 \%$ al internet y tan solo un $2,27 \%$ utilizaba los libros como fuente. En la consulta de si consumen suplementos alimentarios un $55,30 \%$ mencionó que no consumía ningún tipo de suplementos (Tabla 3 ).

Dentro de las principales razones para optar por este estilo de vida un 50,76\% declaró realizarla por principios animalistas, el $21,21 \%$ por beneficios para la salud, en cuanto a lo relacionado con creencias religiosas o por motivos ecológicos tuvo una misma cifra de $9,09 \%$. Entre los aspectos de su alimentación que le resultaba más difícil el $25,76 \%$ contestó asistir a comidas o reuniones sociales, 20,45\% mencionó que ningún aspecto le resulta difícil, el $18,18 \%$ refirió que se le dificulta encontrar alimentos aptos para vegetarianos y la falta de tiempo para preparar sus alimentos (Tabla 3 ).

Tabla 3. Actitudes relacionadas al estilo de vida vegetariano

\begin{tabular}{lll}
\hline Actitudes relacionadas al estilo de vida vegetariano & $\mathbf{n}$ & $\mathbf{\%}$ \\
\hline ¿Cuál fue la razón principal para optar por este estilo de vida? & & \\
Principios animalistas, morales o de protección animal & 67 & $\mathbf{5 0 , 7 6}$ \\
Creencias religiosas/ motivos espirituales & 12 & 9,09 \\
Beneficios para la salud & 28 & 21,21 \\
Razones económicas & 1 & 0,76 \\
Desagrado por el sabor de la carne & 5 & 3,79 \\
Ecológicos & 12 & 9,09 \\
Otro & 7 & 5,30 \\
¿Qué aspecto de su alimentación le resulta más difícil? & & \\
Encontrar alimentos aptos para vegetarianos & 24 & 18,18 \\
Asistir a comidas o reuniones sociales & 34 & $\mathbf{2 5 , 7 6}$ \\
Encontrar alimentos con buen contenido proteico & 2 & 1,52 \\
Salir a comer a restaurantes & 21 & 15,91 \\
Falta de tiempo para preparar sus alimentos & 24 & 18,18 \\
Ningún aspecto me resulta difícil & 27 & 20,45 \\
\hline
\end{tabular}




\begin{tabular}{|c|c|c|}
\hline \multicolumn{3}{|c|}{ Prácticas relacionadas al estilo de vida vegetariano } \\
\hline \multicolumn{3}{|c|}{$\begin{array}{l}\text { ¿Intenta reemplazar las proteínas de origen animal por otras fuentes } \\
\text { proteicas? }\end{array}$} \\
\hline Sí & 119 & 90,15 \\
\hline No & 13 & 9,85 \\
\hline \multicolumn{3}{|c|}{$\begin{array}{l}\text { ¿De dónde obtiene principalmente información nutricional? } \\
\text { (puede marcar más de una opción) }\end{array}$} \\
\hline Nutricionista u otros profesionales de la salud & 16 & 12,12 \\
\hline Círculo familiar y/o social & 5 & 3,79 \\
\hline Internet (blogs, redes sociales, sitios web) & 15 & 11,36 \\
\hline Libros & 3 & 2,27 \\
\hline Autodidacta & 12 & 9,09 \\
\hline Todas las anteriores & 19 & 14,39 \\
\hline \multicolumn{3}{|l|}{ ¿Consume suplementos alimentarios? } \\
\hline Sí & 59 & 44,69 \\
\hline No & 73 & 55,30 \\
\hline
\end{tabular}

\section{DISCUSIÓN}

La proporción de personas que adoptan el estilo de vida vegetariano en relación a la población mundial es escasa, sin embargo, existen cifras que se obtuvieron en base a encuestas, censos y estudios todos ellos realizados por diferentes sociedades vegetarianas y por investigadores de referencia durante los años 2012 y 2016, utilizando en su mayoría métodos on-line para la recolección de los datos ${ }^{(9,14,15)}$. Por su parte en Paraguay, se cuentan con datos escasos sobre vegetarianos, siendo el presente estudio el que pretende obtener mayores datos al respecto.

La totalidad de las encuestas obtenidas fueron realizadas vía on-line mediante difusión por redes sociales, debido a que se trata de un estudio preliminar, dado además el tamaño y dispersión de la población de estudio; tal como en otros estudios realizados por Brignardello et al., Diaz Carmona et al. y Mullee et al. Este método resultó ser más práctico porque la mayoría de los participantes fueron jóvenes, lo que permitió el uso de nuevas tecnologías como las TICs (Tecnologías de la Información y Comunicación) como los formularios de Google Docs, dado que esta población posee mayor acceso y agilidad para el manejo del internet $^{(6,16,17)}$.

De acuerdo con los resultados obtenidos, gran parte de los grupos que respondieron a la encuesta fueron los que llevan el estilo de vida lacto- ovolactovegetariano y en su mayoría del sexo femenino, al igual que en el estudio de Brignardello et al., 2013; en donde un 76\% era lacto- ovolactovegetariano y $81 \%$ de sexo femenino ${ }^{(6)}$.

La edad promedio de los encuestados fue de $28,7 \pm 8.1$ años similar resultado en el estudio

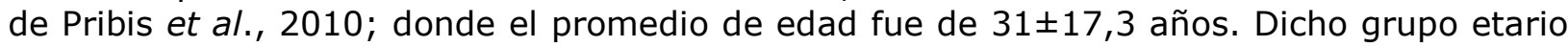
cuenta con mayor acceso al internet, medio por el que se realizó la mayor difusión de la encuesta, además son de cierta manera el grupo que presenta más interés por estilos nuevos y diferentes, se encuentran más expuestos a nuevas tendencias, tienen mayor curiosidad, y defienden nuevas teorías ${ }^{(18)}$.

La distribución geográfica de las personas encuestadas fue predominante del área central en un $87.12 \%$, lo que podría estar condicionado a que pertenecen a una zona que les ofrece mayor facilidad para el acceso a información, se ven más influenciados por modas y culturas nuevas; además de que existe alta disponibilidad de alimentos de origen vegetal con más variedad de locales para adquirir sus alimentos. Resultado similar que arrojó el estudio de Brignardello et al., 2013, quienes hallaron mayor cantidad de vegetarianos en área metropolitana con un $68 \%{ }^{(6)}$.

Así como se menciona en otros estudios sólo un estilo de vida vegetariano adecuadamente equilibrado en cantidad y calidad de alimentos resulta ser beneficioso para la salud; cuando la práctica del mismo conlleva a resultados inapropiados, es probable que se hayan cometido errores dietéticos durante la adopción del mismo ${ }^{(19-22)}$.

El tiempo en el que los participantes han practicado el estilo de vida vegetariano fue variado, personas que iniciaron con una de las variantes dietarías y que realizaron una transición a otra más estricta; otros en cambio se encontraban incursionando en el estilo por un tiempo corto (semanas o meses). Las creencias religiosas de una minoría de los 
participantes se podrían relacionar a una larga duración del estilo de vida, en algunos de ellos de 20 años o más; así como se menciona en el estudio de Hoffman et al., 2013; y en el de Girgis 2015; quienes relacionaron las mismas variables ${ }^{(23,24)}$.

Se evaluaron los conocimientos con base en preguntas referentes a fuentes de los nutrientes críticos y posibles consecuencias que se ven asociados con su bajo aporte, de los cuales se obtuvo que un $41 \%$ presentaba conocimiento insuficiente, $30,03 \%$ suficiente y un $29 \%$ conocimiento regular.

En lo que respecta a las prácticas, $90,15 \%$ de los encuestados respondieron afirmativamente a intentar reemplazar las proteínas de origen animal por otras fuentes proteicas, resultado similar lo obtuvieron Brignardello et al., 2013; donde $89 \%$ de los participantes mencionaron reemplazar las fuentes de proteínas de origen animal por leguminosas y proteínas de soja. La fuente principal de obtención de información fueron los nutricionistas o profesionales de salud $(12,12 \%)$, seguido del internet $(11,36 \%)$; en este caso hubo una diferencia con el estudio de Brignardello et al., 2013; en donde la mayoría, es decir el $45,5 \%$ recurrían a internet ${ }^{6}$. Lo primero pudo deberse a que gran parte de las personas optan por este estilo a causa de motivos relacionados a la salud lo que los obliga a consultar juntamente con un profesional de la nutrición; en cuanto al internet podría asociarse al rango etario de los participantes quienes poseen mayor entrenamiento tecnológico y acceso fácil y rápido de información, lo cual también supone un riesgo de obtener información errónea y no avalada científicamente ${ }^{(6)}$.

La mayoría de los encuestados respondió incursionar al estilo de vida vegetariano por principios animalistas, morales o de protección animal seguido de motivos relacionados a la salud; al igual que en otros estudios que también investigaron las razones de optar por el estilo de vida vegetariano ${ }^{(18,23,25)}$. Entre los aspectos que le resultaron más difíciles al seguir este estilo de vida, se mencionaron el sentimiento de discriminación y asistir a comidas o reuniones sociales los cuales fueron el mayor porcentaje de respuestas obtenidas. Esto puede deberse a que actualmente este estilo no es muy practicado en Paraguay, lo que lleva a que las personas lo consideren como un estilo limitante y hasta imposible de practicar en el ambiente carnívoro en el que vivimos.

En lo referente al conocimiento sobre el estilo de vida vegetariano, fue predominante el conocimiento regular y suficiente lo que difiere con el estudio de Brignardello et al., 2013(6), lo cual podría deberse a que gran parte de los encuestados contaba mayoritariamente con un nivel educativo terciario, lo cual coincide con otros estudios realizados; seguido del nivel secundario $^{(16,17,23)}$. Además, participaron profesionales de la salud de diferentes áreas, cuya formación podría propiciar al mayor conocimiento sobre el tema en cuestión.

En conclusión, los encuestados presentaron conocimiento regular seguido de insuficiente en lo que respecta a su estilo de vida y conocimiento insuficiente sobre nutrientes críticos. Respecto a sus prácticas, gran parte de ellos reemplazan las proteínas por las de origen vegetal obteniendo información de diversas fuentes. Actitudinalmente creen que su alimentación favorece a su salud e imagen corporal y la recomiendan; habiendo sufrido discriminación en algún momento de su vida mediante críticas o burlas hacia su estilo de vida.

El presente estudio es el primero en cuanto a conocimientos, prácticas y actitudes que se realiza a nivel país pretendiendo obtener cifras de vegetarianos; lo que resulta ser innovador y desafiante debido a la población elegida. Además de que se convierte en una base para la realización de futuras investigaciones de manera a seguir conociendo aún más sobre esta población que se encuentra en aumento.

También la utilización de los medios tecnológicos favoreció a la captación eficaz de los sujetos de estudio facilitando la realización de la encuesta tanto para los encuestados como para las investigadoras; en cuanto a privacidad y agilidad.

\section{REFERENCIAS BIBLIOGRÁFICAS}

1. Farran A, Illan $M$, Padró L. Dieta vegetariana $y$ otras dietas alternativas. Pediatría Integral. 5th ed. Sociedad Española de Pediatría Extrahospitalaria y Atención Primaria (SEPEAP); 2015: 313-23.

2. Gallo D, Manuzza M, Echegaray N, Montero J, Munner M, Rovirosa A et al. Grupo de trabajo alimentos de la sociedad argentina de nutrición-
Alimentación Vegetariana. Buenos Aires; 2014: 1-35.

3. Martínez M, Vázquez Z. Patrón de Dieta Pro-Vegetariana y Mortalidad general. Rev Chil Nutr. 2014; 41 (4): 367-71.

4. Bravo P, Ibarra J, Paredes M. Compromiso Neurológico y hematológico por déficit de vitamina B12 en lactante hijo de madre 
vegetariana. Caso Clínico. Rev Chil Pediatr 2014; 85 (3): 337-43.

5. Glick M, Yeh $M$. The Health Advantage of a Vegan Diet: Exploring the Gut Microbiota $y$ Connection. Nutrients. 2014; 6: 4822-38.

6. Brignardello $J$, Heredia $L$, Ocharán $M$, Duran S. Conocimientos Alimentarios vegetarianos y veganos Chilenos. Rev Chil Nutr 2013; 40(2): 129-33.

7. Cornejo V. La Moda de la Dieta Vegetariana: sus efectos en la salud. Revista del Instituto de Nutrición y Tecnología de los Alimentos de la Universidad de Chile. 2014; 109(7):1266-82.

8. Orellana L, Sepulveda J, Denegri $M$. Significado Psicologico de comer carne, vegetarianismo y alimentación saludable en estudiantes universitarios a partir de redes semánticas naturales. Rev Mex Trastor Aliment 2013: 15-22.

9. Leitzmannc. Vegetarian Nutritión: Past, Present, Future. Am J Clin Nutr 2014; 100 (Suppl): 496S-502S.

10. Ministerio de Educación y Ciencia. Evaluación del Aprendizaje. Asunción: MEC; 2009.

11. De la Evaluación. [base de datos en Internet]; [agosto de 2017]. [aproximadamente 3 pantallas].

Disponible

en:

http://www.agr.una.py/descargas/reglamentos/ DE_LA_EVALUACION.pdf.

12. Navarro LE, Vargas Morant R. Conocimientos, actitudes y prácticas sobre SIDA en adolescentes escolarizados. Revista Salud Uninorte. 2004; 19:14-24.

13. Díaz Vélez C, Manrique González L, Galán Rodas E, Apolaya Segura M. Conocimientos, actitudes y prácticas en investigación de los estudiantes de pregrado de facultades de medicina del Perú. Acta Med Per. 2008; 25(1): 9-14.

14. Estadísticas sobre vegetarianismo y veganismo. [base de datos en Internet].2011/12-[acceso 10 de agosto de 2017]. Disponible en: http://www.respuestasveganas.org/2011/12/en cuestas-sobre-veganismo.html.
15. Chile: resultados del primer censo vegetariano. [base de datos en Internet] Uribe R., I.; 2013-[acceso 10 de agosto de 2017]. Disponible en: http://www.vegetarianoschile.cl/chileresultados-del-primer-censo-vegetariano/. 16. Díaz Carmona E. Perfil del vegano/a activista de liberación animal en España. Rev Esp Invest Sociol 2012; 175-88.

17. Mullee $A$, Vermeire $L$, Vanaelst $B$, Mullie $P$, Deriemaeker $P$, Leenaert $T$ et al. Vegetarianism and meat consumption: A comparison of attitudes and beliefs between vegetarian, semivegetarian, and omnivorous subjects in Belgium Appetite 2017; 114:299-305.

18. Pribis P, Pencak R, Grajales T. Beliefs and Attitudes toward Vegetarian Lifestyle across Generations. Nutrients. 2010; 2:523-31.

19. Santana Vega C, Carbajo Ferreira A. Dieta vegetariana. Beneficios y riesgos nutricionales. Form Act Pediatr Aten Prim 2016; 4(9):161-67. 20. Pilis W, Stec K, Zych M, Pilis A. Health benefits and risk associated with adopting a vegetarian diet. Rocz Panstw Zakl Hig. 2014; 65(1):9-14.

21. Teixeira R, Molina M, Zandonade E, Mill J. Risco cardiovascular em vegetarianos e onívoros: um estudo comparativo. Arq Bras Cardiol. 2007; 89(4):237-44.

22. Cayllante Cayllagua, J. Vegetarianismo. Revista de Actualización Clínica. 2014; 42: 2195-7.

23. Hoffman $S$, Stallings $S$, Bessinger $R$, Brooks G. Differences between health and ethical vegetarians. Strength of conviction, nutrition knowledge, dietary restriction, and duration of adherence. Appetite. 2013; 65:13944.

24. Girgis E. The Reasons for Consuming a Vegetarian Diet in Lebanon and the Survey of Its Health Impacts. World Family Medicine Journal/Middle East Journal of Family Medicine. $2015 ; 13(1): 4-14$.

25. Rothgerber $\mathrm{H}$. A comparison of attitudes toward meat and animals among strict and semi-vegetarians. Appetite. 2014; 72:98-105. 